\title{
Prediction of Breast Tissue and Plastic Reconstruction in Different Positions by Finite Element Method
}

\author{
Dongwei Dong ${ }^{1}$, Tingchun $\mathrm{Shi}^{1{ }^{1,},}$, Jie Luan ${ }^{2}$ and Xiuyan Yue \\ ${ }^{1}$ College of Life Information Science \& Instrument Engineering, Hangzhou Dianzi University, Hangzhou, P.R. China \\ ${ }^{2}$ Department of Aesthetic and Plastic Breast Surgery, Plastic Surgery Hospital, Peking Union Medical College, Chinese Academy \\ of Medical Sciences, No. 33 Badachu Road, Shijingshan District, Beijing, P.R. China \\ ${ }^{3}$ Hangzhou Dianzi University Library, Hangzhou Dianzi University, Hangzhou, P.R. China \\ *Corresponding author
}

\begin{abstract}
Breast augmentation surgery is a way to improve the beauty of female body shapes. Based on magnetic resonance imaging (MRI) images of patient's breast providing real simulation data for breast augmentation surgery, this study investigated the effects of two different shapes of implants with the same size on the breast augmentation, and the impact on the breast deformation in lying and standing-up position. After the numerical simulation, the difference of the maximum total deformation between the anatomical prosthesis and the normal breast was $19.478 \mathrm{~mm}$ in the standing-up position, which was $2.75 \%$ less than the total deformation of the round prosthesis. In the lying position, the difference between the round prosthesis and the normal breast was $20.591 \mathrm{~mm}$, which was $1.26 \%$ less than that of the anatomical prosthesis. The results indicated that the anatomical implanted breast in the standing position had the smaller total deformation caused by gravity, which is more conducive to the maintenance of the shape and more firm. Similarly, round prosthesis is superior to anatomical in lying position. The method proposed in this paper can reasonably predict the degree of deformation of the breast at different postures and reveal the potential capacity of visualization and prediction of the finite element(FE) simulation.
\end{abstract}

Keywords-augmentation mammoplasty; silicone prosthesis; finite element method; shape prediction

\section{INTRODUCTION}

With the aesthetic improvement, women's demand for breast augmentation has been growing. Breast augmentation was the most normally performed plastic surgery procedure in 2011 with 300,000 women in the United States, a 45\% increase from 2000 [1][2].Since the early 1960s, Cronin et.al [3] used silicone as implants at the first time, which causes breast augmentation surgery popularized rapidly. The surgery enlarges and reshapes the shape of the breast by placing an implant, which is covered by an elastic tissue inside the breast.

Breast is a complex organ that is composed of soft tissues (fat, gland and skin) [4], whose proportions and properties between individuals differ according to factors such as age or health status. Meanwhile, different sizes and types of implants on the breast has increased the difficulty of predicting breast deformation with the impact of gravity in various positions.

In a certain degree of accuracy, the results of breast surgery can be predicted mostly relying the skills of surgeons. However, the reality shows person's aesthetic is different from others, and there are always discrepancies between doctors and patients. millions of women are asking for revision surgery because breast deformities differ from their expectations after implantation.

Before surgery, the clinician must collect the necessary information about predictions of the patient's breast deformities to develop preoperative planning. The situation of the breast in standing-up position estimated via the data typically collected by anthropometric measurements [5], as well as the position, type, size and shape of the implant.

Based on the medical images obtained from patients in real clinical cases, this paper described a method, which is that FE model simulated the deformation of breast affected by gravity as the human body in different positions. At the same time, after various types of prostheses being implanted in the breast, the comparison of the degree of deformation impacted by gravity was studied. The goal of such a simulation is to predict the final shape of the breast in the standing-up position of the patient after placing the prosthesis before breast augmentation surgery.

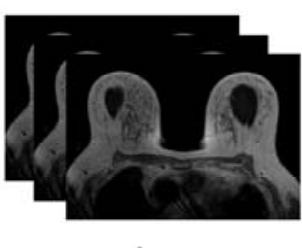

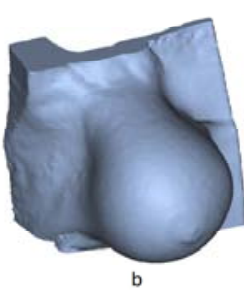

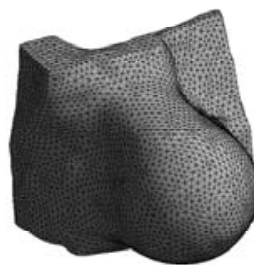

c

(a) different MRI slices. (b) geometric model of the patient's breast. (c) anteroposterior view of the FE mesh

FIGURE I. FE MODEL GENERATION OF THE PATIENT'S RIGHT BREAST KNEELING ON FOUR IN THE PROSTRATE POSITION. 


\section{MATERIALS AND METHODS}

In this study, a 58-year-old Asian woman without mammary gland disease was chosen to be a clinical case of augmentation mammoplasty, from whom informed consent was obtained. An anatomical silicone gel implant and a round silicone gel implant, with the same volume, surface area, height, were used as fillers in the posterior side of the right breast, and the virtual FE planning model was proposed in this paper to simulate the deformation of breast which was impacted by the gravity in different positions.

\section{A. FE model Generation}

The FE model of the breast was constructed by utilizing magnetic resonance imaging (MRI) images [6][7] of the patient. When the images were collected, the patient was kneeling on four in the prostrate position, keeping the body fixed and dropping the breast naturally (Fig. 1a). The images with 128 slices were obtained by a Siemens / Aera MRI scanner with a $384 *$ 384-pixel resolution; $1.65 \mathrm{~mm}$ slice thickness and pixel size $0.938 \mathrm{~mm} * 0.938 \mathrm{~mm}$ in-plane. The images were saved in DICOM format and loaded into MIMICS 10.01 software [8]. The software automatically applies thresholds to separate skin, fat, breast, and muscle, as well as obtaining associated tissue by manual segmentation. Finally, the 3D geometry model (Fig. 1b), including fat and gland, was generated. The geometric model of the patient was exported to Ansys 15.0 [9] to generate an FE model. A grid consisting of 131,922 linear hexahedral elements and 24,662 nodes representing breasts was obtained using automated meshing techniques (Fig. 1c). Subsequently, the grid convergence test is performed, and the suitability of the selected mesh size was checked.

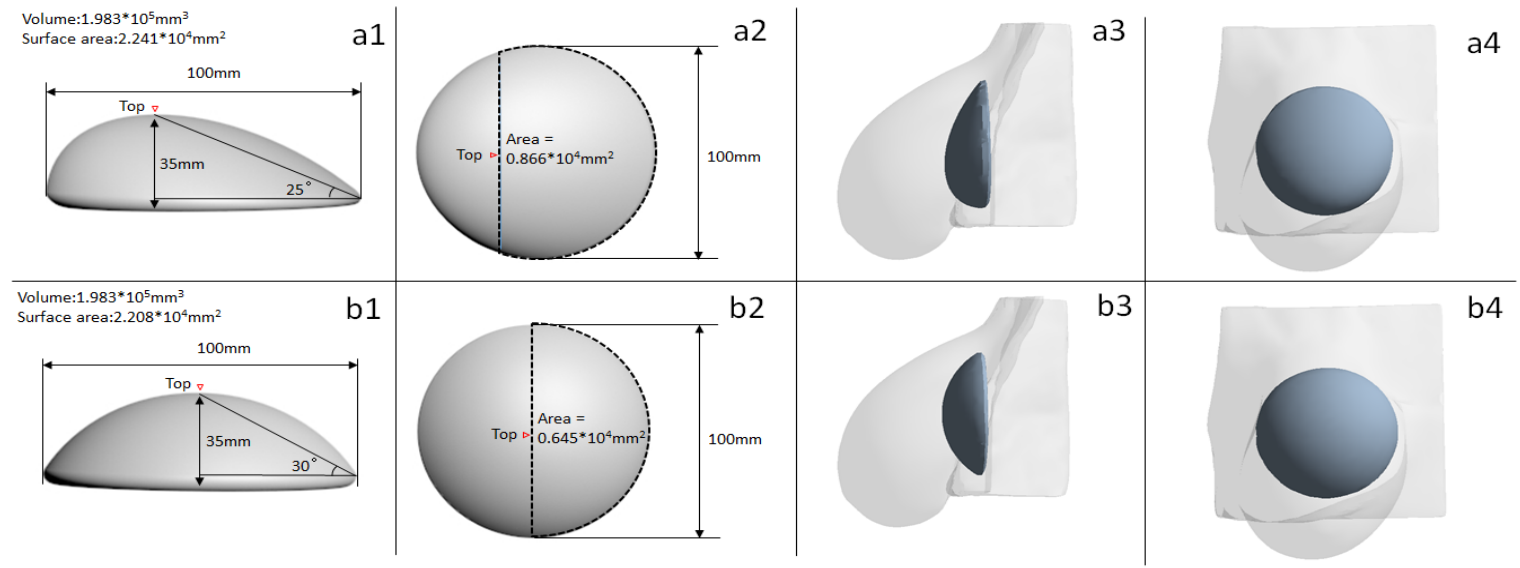

FIGURE II. PARAMETERS AND PLACEMENT OF ANATOMIC AND ROUND PROSTHESES

\section{B. Simulation of Prosthesis Implantation}

The posterior mammary gland space is one of the most commonly used positions for breast implants, and which is a space between the breast and the muscle after implantation of the silica gel prosthesis. In the condition of the same size of breasts, authors study mainly the deformation of the breast with the prosthesis implantation and without it. The two prosthesis replaced the fat tissue near muscle in the same place. (Fig. II)

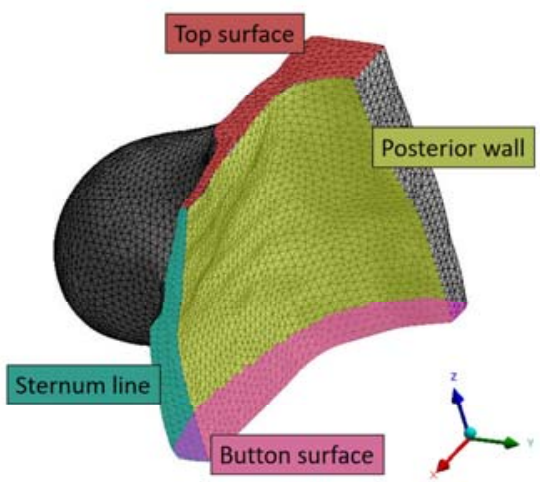

FIGURE III. POSTEROANTERIOR VIEWS SHOWING COLOR-CODED FACES FROM DIFFERENT ANATOMICAL LOCATIONS WHERE FIXED DISPLACEMENT

\section{Material Parameters \& Boundary conditions}

On account of the mean values of Young's modulus, Density, Poisson's ratio [10][11][12] for normal breast tissues and silicone gel $\left(\mathrm{E}_{\text {fat }}=3.24 \mathrm{kPa}, \mathrm{E}_{\text {gland }}=3.25 \mathrm{kPa}, \mathrm{E}_{\text {silicone }}=250\right.$ $\mathrm{kPa} ; \rho_{\text {fat }}=\rho_{\text {gland }}=9.05 * 10^{-7} \mathrm{~kg} / \mathrm{mm}^{3}, \rho_{\text {silicone }}=1.08 * 10^{-6}$ $\mathrm{kg} / \mathrm{mm}^{3} ; v_{\text {fat }}=v_{\text {gland }}=v_{\text {silicone }}=0.49$ ). Therefore, fat and gland were considered as a homogenized breast tissue in the present work [13][14].

In the FE model, the proper definition of the boundary conditions is more important than the material properties of the resulting accuracy [15]. To properly simulate the deformation of the breast, the fixed displacement boundary conditions were applied to certain locations in the FE model. The rear of the breast, fully attached to the muscle tissue, was set because the muscle tissue is almost motionless when the impact of postural changes. The breast tissue in front of the sternum line was also constrained because the sternum was unmovable. The top and bottom surfaces, which respectively constrain the upper and lower portions of the breast, and couldn't change the accuracy of the result due to the far distance with breast, were also stationary. Finally, the binding conditions of the implanted silica gel and its surrounding tissue are no separation, and which allowed the existence of a small amount of friction free slip. (Fig. III) 


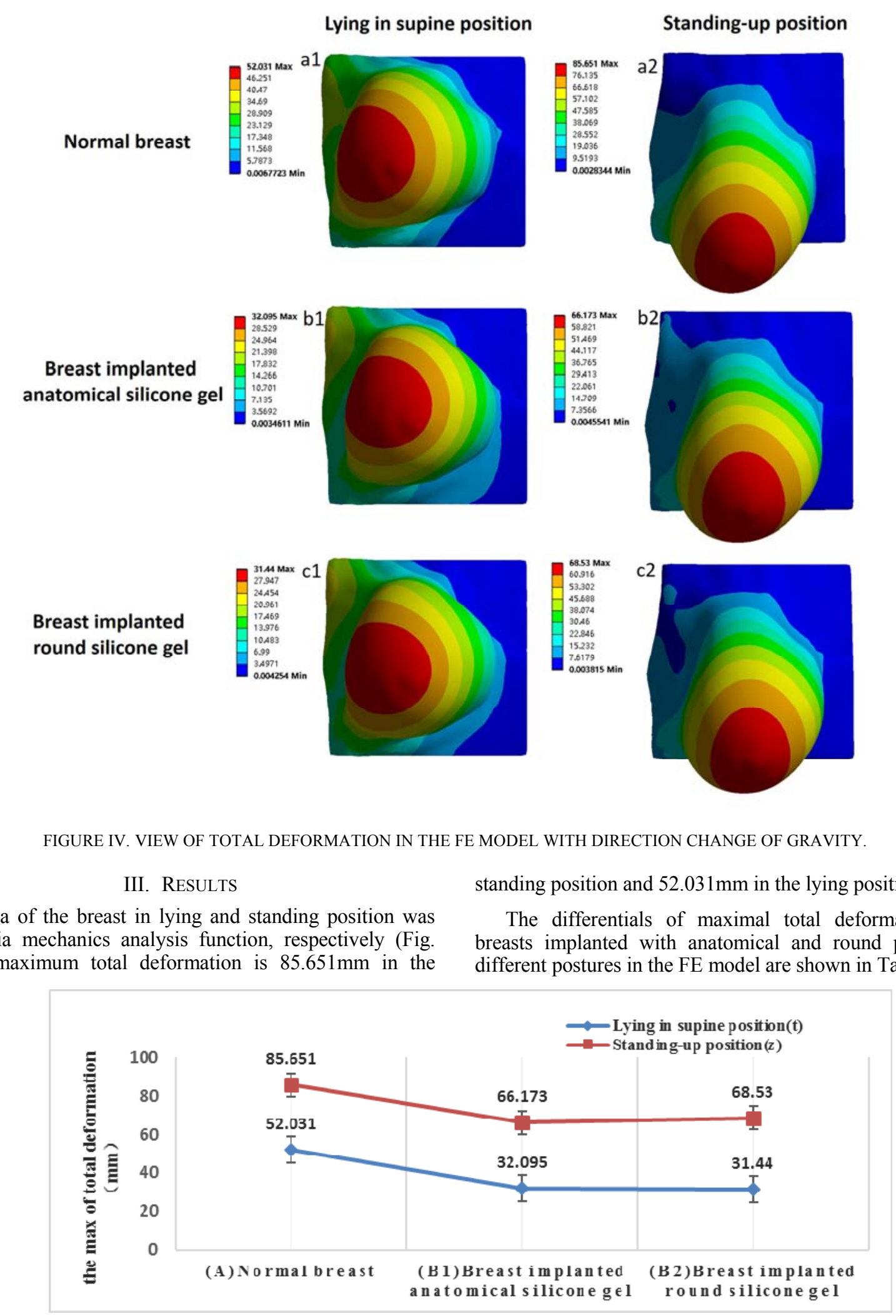

FIGURE V. TENDENCY OF TOTAL DEFORMATIONS OF BREASTS IMPLANTED VARIOUS TYPES OF PROSTHESES. 
TABLE I. DIFFERENCE OF MAXIMAL DEFORMATIONS BETWEEN BREASTS AND AUTOLOGOUS BREASTS OF DIFFERENT IMPLANTED PROSTHESES IN DIFFERENT POSITIONS

\begin{tabular}{|c|c|c|}
\hline $\begin{array}{c}\text { differentials of maximal } \\
\text { total deformation }\end{array}$ & $\begin{array}{c}\text { Lying in supine } \\
\text { position }\end{array}$ & $\begin{array}{c}\text { Standing-up } \\
\text { position }\end{array}$ \\
\hline $\mathrm{A}$ and B1 $(\mathrm{mm})$ & 19.936 & 19.478 \\
\hline $\mathrm{A}$ and B2 $(\mathrm{mm})$ & 20.591 & 17.121 \\
\hline
\end{tabular}

The degree of reduction in the maximum amount of deformation of the normal breast:

$$
\operatorname{Ln} x=\frac{D n x}{A x}=\frac{A x-B n x}{A x} \times 100 \%
$$

$$
\begin{aligned}
& \mathrm{L} 1 \mathrm{t}=38.32 \% ; \mathrm{L} 1 \mathrm{z}=22.74 \% \\
& \mathrm{~L} 2 \mathrm{t}=39.57 \% ; \mathrm{L} 2 \mathrm{z}=19.99 \%
\end{aligned}
$$

Comparing the situations of the breast with silica gel and without implantation, we found that the total deformation was reduced to $38.32 \%$ (Anatomical) and $39.57 \%$ (Round) of the normal breast's result in lying position, respectively. While the deformation decreased by $22.74 \%$ (Anatomic) and $19.99 \%$ (Round) in the same position. The reduction of the total deformation indicated that the degree of breast deformation after implantation of silicone was reduced and the appearance of breast became more upright and beautiful, which was in accord with the desired purpose of implant breast augmentation surgery.

\section{DISCUSSION}

In this paper, the authors present an FE-based methodology based on patient-specific, where the breast deformation caused by gravity is simulated using FE theory. The ultimate goal of this paper is to propose a direct method for predicting the deformation of the breast at different postures.

In this study, we simplified the tissue structure of the breast and researched its maximum total deformation in the standing up and lying positions. The results showed that the total deformation of the breast in the standing up position was much greater than that in the lying position, which indicated that the breast constraint was better when the human breast muscle acts as a constraining surface in the lying position. Breast deformation in the simulation deformation trends was in line with the reality of the human condition, showing that the method of experiments is feasible.

Compared the data of total deformation of the breast with implanted and non-implanted prosthesis, the silicone prosthesis plays a great role in maintaining morphology and appearance (more upright) of the breast.

At the same time, the aesthetic evaluation standard of breast plasticization takes into account the standing position, so the smaller morphology of breast augmentation changes in the standing-up position, and the postoperative evaluation is much higher. Compared the anatomical prosthesis and round prosthesis, both of which almost were with the same volume and area, and simulated to be implanted into the breast, respectively. The study found that anatomical prosthesis for the breast maintenance effect is better than the round prosthesis in standing up position.

Although simplifying the geometry and material of the breast, the authors suggest that the proposed and verification methods are straightforward, and which can simulate the deformation of the breast at different postures. However, there is a need to refine this approach further and to subdivide the tissue of the breast to more precisely rebuilt the patient-specific FE model [16]. Considering the simplicity of this approach, the development of further FE models for similar situations may be a valuable tool to help clinicians plan and predict the outcome of breast surgery.

\section{ACKNOWLEDGMENT}

This study was supported by the National Natural Science Foundation of China (61272389).

\section{REFERENCES}

[1] Rohrich, R.J., The increasing popularity of cosmetic surgery procedures: a look at statistics in plastic surgery. Plastic \& Reconstructive Surgery, 2000. 106(6): p. 1363-5.

[2] Endsley, S., et al., American Society of Plastic Surgeons. CRC Press Featured Authors, 2010.

[3] Van, Z.D. and O. Heymans, Breast implants. A review. Acta Chirurgica Belgica, 2004. 104(2): p. 158-65.

[4] Johns, P.C. and M.J. Yaffe, X-ray characterisation of normal and neoplastic breast tissues. Physics in Medicine \& Biology, 1987. 32(6): p. 675-95.

[5] Qiao, Q., G. Zhou and Y. Ling, Breast volume measurement in young Chinese women and clinical applications. Aesthetic Plastic Surgery, 1997. 21(5): p. 362-368.

[6] Lee, H.Y., K. Hong and E.A. Kim, Measurement protocol of women's nude breasts using a 3D scanning technique. Applied Ergonomics, 2004. 35(4): p. 353-9.

[7] Tepper, O.M., et al., Virtual 3-dimensional modeling as a valuable adjunct to aesthetic and reconstructive breast surgery. American Journal of Surgery, 2006. 192(4): p. 548-51.

[8] Tepper, O.M., et al., Virtual 3-dimensional modeling as a valuable adjunct to aesthetic and reconstructive breast surgery. American Journal of Surgery, 2006. 192(4): p. 548-51.

[9] Moaveni S. Finite Element Analysis: Theory and Application with Ansys[M]. Prentice Hall, 1999.

[10] Samani, A., J. Zubovits and D. Plewes, Elastic moduli of normal and pathological human breast tissues: an inversion-technique-based investigation of 169 samples. Physics in Medicine \& Biology, 2007. 52(6): p. 1565-76.

[11] Kuhlmann, M., et al., Mechanical model of the breast for the prediction of deformation during imaging. Medical Engineering \& Physics, 2012. 35(4): p. 470-478.

[12] Rynkevic, R., et al. Implant shape influence on the mechanical behavior of breast implants. in Bioengineering. 2013.

[13] Del Palomar, A.P., et al., A finite element model to accurately predict real deformations of the breast. Medical Engineering \& Physics, 2008. 30(9): p. 1089-1097.

[14] Roose, L., et al., Validation of different soft tissue simulation methods for breast augmentation. International Congress, 2005. 1281: p. 485-490.

[15] Tanner, C., et al., Factors influencing the accuracy of biomechanical breast models. Medical Physics, 2006. 33(6): p. 1758-69.

[16] Bakic, P.R., et al., Mammogram synthesis using a 3D simulation. I. Breast tissue model and image acquisition simulation. Medical Physics, 2002. 29(9): p. 2131-2139. 